\title{
Risk factors for canine cognitive dysfunction syndrome in Slovakia
}

Stanislav Katina ${ }^{1 \dagger}$, Jana Farbakova ${ }^{2 \dagger}$, Aladar Madari ${ }^{2+}$, Michal Novak $^{3}$ and Norbert Zilka ${ }^{3,4^{*}}$

\begin{abstract}
Background: Increasing prevalence of cognitive impairment in an aging canine population poses a serious health problem. Identifying risk factors, which may influence the onset of cognitive decline, is becoming increasingly important. Here we investigated whether age, sex, weight, nutrition, dogs' housing and reproductive state were associated with increased risk of canine cognitive dysfunction syndrome (CCDS) in Slovakia.

Results: Age was associated with cognitive decline and nutrition emerged as a significant predictor variable. Dogs fed controlled diets had 2.8 times lower odds of developing CCDS when compared with dogs fed uncontrolled diets. Sex, weight, reproductive state and dogs' housing were not significantly associated with cognitive decline. Further, the prevalence of CCDS was similar in both small and medium/large sized dogs aged 8-11 years, but differed in dogs at an age of 11-13 years.
\end{abstract}

Conclusion: Age was found to be the most prominent risk factors of CCDS. Nutrition may influence the cognitive state of dogs. This finding suggests that nutritional interventions may modify canine cognitive functions.

Keywords: Canine cognitive dysfunction syndrome, Cognitive decline, Risk factors, Prevalence, Epidemiology, Nutrition

\section{Background}

Canine cognitive dysfunction syndrome (CCDS) is a complex of behavioural symptoms in aged dogs characterised by deficits in learning, memory, perception, awareness and by impairment of social interactions and sleeping patterns [1]. Improvements in nutrition, elimination of most infectious diseases, better hygiene and adoption of antibiotics and vaccines have increased the life expectancy of dogs. The immediate consequence of the extended life span is an increased number of aged dogs. It has been estimated that there are more than 45 million dogs around 7-years-old in USA and Europe [2]. Because ageing is the main risk factor of CCDS, aged dogs likely represent a large population with a great risk of developing CCDS [3]. Indeed, Neilson et al. [4] showed

\footnotetext{
*Correspondence: norbert.zilka@savba.sk

†'Stanislav Katina, Jana Farbakova and Aladar Madari contributed equally to this work

${ }^{4}$ Institute of Neuroimmunology, No., Dvorakovo Nabrezie 45, Bratislava, Slovak Republic

Full list of author information is available at the end of the article
}

that the prevalence of CCDS was $28 \%$ in 11-12 years old dogs and $68 \%$ in 15-16 years old dogs. Similarly, Azkona et al. [5] demonstrated that $22.5 \%$ of dogs older than 9 years displayed cognitive impairment.

As a result of the increasing number of cognitively impaired dogs, increasing emphasis is being placed on management and therapy of an age-related canine cognitive impairment [3]. Thus identifying modifiable risk factors and developing preventive strategies for CCDS represent important goals. Currently, there are only limited data available about the risk factors of CCDS, as only a few studies dealing with the risk factors of CCDS have been conducted over the last decade. A study carried out in Spain suggested an association between sex, size, reproductive state and cognitive function of aged dogs [5]. That study showed that females and neutered dogs were significantly more affected than males and intact dogs. Moreover, small breeds had greater odds of showing age-related cognitive impairment than medium or large sized dogs, although weight was not a statistically significant predictor variable. The major drawback of the 
study lied in structured phone interviews that did not take into account the subjective evaluation of pet owners. Similarly, Hart et al. [6] demonstrated that sexually intact male dogs were significantly less likely than neutered dogs to progress from mild impairment to severe impairment. Another study, conducted in Denmark, showed that neither sex nor weight correlated with cognitive decline [7]. Contradictory results are likely caused by different methodologies used for detection of canine cognitive decline.

The aim of the present study was to test several variables, age, sex, reproductive status, bodyweight, dogs' housing and nutrition in order to identify possible risk factors for CCDS.

\section{Methods}

\section{Animals}

Study animals were identified among all dogs visiting the Small Animal Veterinary Clinic of the University of Veterinary Medicine and Pharmacy in Kosice, Slovak Republic for regular vaccination, parasite treatments and various health complaints. The age of study dogs ranged from 8 to 18 years (mean $=11$ years), there were 116 males and 99 females of different breeds, body weights ranged from 2.3 to $55 \mathrm{~kg}$ (mean $=19.6 \mathrm{~kg}$ ) and reproduction state was neutered males $(n=88)$, neutered females $(n=79)$; intact males $(\mathrm{n}=25)$, and intact females $(\mathrm{n}=23)$.

\section{Haematology and biochemistry}

An integral part of the diagnosis was haematological and biochemical blood tests. The following haematological variables were analysed by IDEXX ProCyte Dx ${ }^{\circledR}$ Hematology Analyzer: HCT, RBC, HGB, MCV, MCH, MCHC, red cell distribution width (RDW), reticulocytes (absolute number and percentage), WBC, neutrophils, lymphocytes, monocytes, eosinophils, and basophils (number and percentage), platelets [number, MPV, platelet distribution width (PDW) and PCT], band neutrophils (when presence suspected) and nRBCs (nucleated red blood cells, when presence suspected). Biochemical blood parameters were analysed by Cobas $C 111$ analyzer (Roche). These variables included ALT, AST, ALP, pAMS, LIP, Crea, UREA, Glu, Chol, TP, Alb, Ca, P, Mg, NH3, K, Na, Cl. All dogs included in this study were assessed by neurological examination, orthopaedic, radiology, ultrasound and ECG examination, as well as by blood and urine analyses. Eighty-five dogs were excluded from the study because of other medical causes interfering with cognitive decline such as blindness, deafness, diabetes mellitus, cushing syndrome, urinary tract infection, incontinence of urine or faeces, cardiological patients, head trauma and other disease conditions.

\section{Cognitive evaluation}

Behavioural investigation included observation of geriatric dogs by a veterinary clinician and collection of information provided by pet owners. The investigator was pro-active in asking about behavioural abnormalities to identify even subtle signs that often go unrecognized by pet owners. Data collected by using questionnaire was important for calculation of the final score. The questionnaire also included basic information about dog characteristics and lifestyle variables such as sex, age, weight, reproductive state, dog's housing and type of diet. The composite scale-CAnine DEmentia Scale (CADES) used in this study was adapted and modified from the questionnaires proposed by Osella, et al. [8] and Salvin et al. [9]. It contained 17 items distributed into four domains (spatial orientation, social interactions, sleepawake cycles and house soiling) related to changes in dog's behaviour. The value of each item corresponded to the frequency of abnormal behaviour. We used a 5-point scale for easy evaluation of behaviour: 0-abnormal behaviour of the dog had never observed; 2-abnormal behaviour of the dog was detected at least once within the last 6 months; 3-abnormal behaviour appeared at least once per month; 4-abnormal behaviour was seen several times per month; 5-abnormal behaviour was observed several times a week. The score from each domain was added up to obtain a final quantitative score that reliably reflected the qualitative evaluation of cognitive decline. We validated CADES as a screening tool for CCDS [10]. Dogs were divided into two subgroups: dogs with no or very mild cognitive impairment (MiCI) and dogs with an advanced cognitive decline. The first group consists of cognitively normal dogs (NA; CADES score $0-7$ ) and dogs with MiCI (CADES score 8-23). The second group consists of dogs with moderate cognitive impairment (MoCI; CADES score 23-44) and dogs with severe cognitive impairment (CADES score higher than 44).

\section{Prevalence calculation}

The prevalence of CCDS in three age groups was calculated. All tested dogs were divided into age groups based on estimated life expectancies [11]: short-lived, 6-11 years; medium-lived, 11-13 years and long-lived, $>13$ years, as proposed by Salvin et al. [9]. The prevalence was calculated by dividing the number of dogs in each tested group (NA, MiCI, MoCI, CD) by the number of individuals examined in each age category.

\section{Data analysis}

Statistical analyses were performed with $\mathrm{R}$ software [12]. Univariable logistic regression analysis (two-sample $Z$-test of $\log$ odds) [13] was used to assess relationships between cognitive impairment as dependent variable (moderate and severe CCDS vs normal ageing and mild cognitive impairment) and risk factors such as sex (males vs females), reproductive state (neutered vs entire dogs), food (uncontrolled-scrabs, commercial dry or wet food low quality or mix of different kind of food vs controlled 
diet-commercial dry or wet food for specific breed, age or life stages-obese, neutered, intact, working dogs), dog's housing (outside-dogs spending much of their time outside the house vs inside-dogs spending much of their time inside the house or flat) and weight (under $15 \mathrm{~kg}$ vs over $15 \mathrm{~kg}$, under $15 \mathrm{~kg}$ means smaller or equal than $15 \mathrm{~kg}$ in the paper) as independent variables. All age and weight related intervals used in the text are open from left and closed from right, e.g. 11-13 years means greater than 11 and smaller or equal than 13 (except for the first in the range, which is closed from both sides, e.g. 8-11 years means greater or equal than 8 and smaller or equal than 11). The risk was considered to be positive with respect to cognitive impairment when the estimate of odds ratio (OR) was greater than one, and negative when OR was less than one. Additionally, Wald $95 \%$ empirical confidence intervals for OR were calculated. To test correlation between age (in years) and composite CADES score, one-sample Fisher Z-test of zero correlation was used. Additionally, Wald $95 \%$ empirical confidence intervals for Pearson product-moment correlation coefficient were calculated. All of the null hypotheses were tested against two-sided alternatives on significance level $\alpha=0.05$. Finally we also used saturated additive multivariable logistic regression model with interaction (of sex and reproductive state) of the form-cognitive impairment $\sim$ sex + reproductive state + diet + housing + weight + (sex: reproductive state), where the log odds were tested similarly as in univariate analyses equivalent to univariable logistic regression model.

\section{Results}

\section{Correlation of age and CCDS}

First, we focused on relationship between advancing age and CADES score reflecting degree of cognitive impairment. There was positive correlation between both advancing age and CADES score (estimate of Pearson product-moment correlation coefficient $r=0.662, t=12.895$, $d f=213, P<0.0001,95 \%$ CI: (0.580, 0.731); Fig. 1$)$.

\section{Association between nutrition and CCDS}

For the study of putative risk factors, the dog population with a slightly uneven sex ratio [54\% males, $46 \%$ females] was classified as follows: weights-small size breeds (less than or equal to $15 \mathrm{~kg} ; 112 \mathrm{dogs}$ ) or medium/ large size breeds (over $15 \mathrm{~kg} ; 103$ dogs), nutrition-controlled diets (113 dogs) or uncontrolled diets (102 dogs), dogs' housing-outside (78 dogs) or inside (137 dogs) and reproductive status - neutered (167 dogs) or entire dogs (48 dogs); see Table 1.

Univariable analyses demonstrated that there was a significant association between nutrition and CCDS $(P<0.001)$. Dogs fed with controlled diet displayed 2.8 times lower odds of CDS when compared to dogs fed

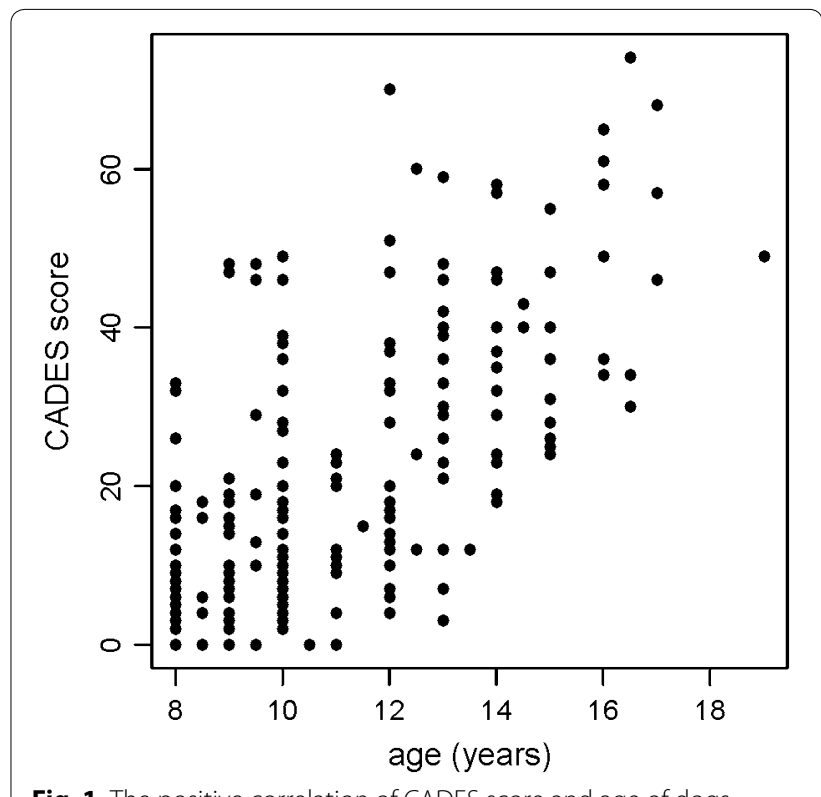

Fig. 1 The positive correlation of CADES score and age of dogs

uncontrolled diets $(\mathrm{OR}=2.8)$. Sex $(P=0.11)$, weight $(P=0.14)$, reproductive status $(P=0.32)$ and dogs' housing $(P=0.42)$ were not significant at the univariable level (Table 2; Fig. 2). Multivariable analyses showed similar results. Sex, reproductive state, housing, weight, and interaction of sex and reproductive state were all ruled out from saturated model and the only statistically important variable is diet $(\mathrm{OR}=2.8, P<0.001)$.

\section{The prevalence of CCDS increased with age}

We found that the prevalence of fully developed CCDS $(\mathrm{MoCI}+\mathrm{CD})$ was $13 \%$ (7 dogs, below $15 \mathrm{~kg}$ ) and $16 \%$ (12 dogs, over $15 \mathrm{~kg}$ ) in the age period 8-11 years, $41 \%$ (11 dogs, below $15 \mathrm{~kg}$ ) and $65 \%$ (13 dogs, over $15 \mathrm{~kg}$ ) in the age period 11-13 years and $87 \%$ (26 dogs, below $15 \mathrm{~kg}$ ) and $100 \%$ (9 dogs, over $15 \mathrm{~kg}$ ) in the age over 13 years. Further, $52 \%$ (28 dogs, below $15 \mathrm{~kg}$ ) and $42 \%$ (31 dogs, over $15 \mathrm{~kg}$ ) of 8-11 years old dogs and $37 \%$ (10 dogs, below $15 \mathrm{~kg}$ ) and $35 \%$ (7 dogs, below $15 \mathrm{~kg}$ ) of 11-13 years old dogs were classified as mildly affected (MiCI). Prevalence of CCDS was similar in both small and medium/large sized dogs in the age period 8-11 years (13 vs $16 \%$ ), and differed in older dogs, $11-13$ years old (41 vs $65 \%$ ); see Table 3; Fig. 3. In dogs older than 13 years, we did not find any cognitively intact dog.

\section{Discussion}

The cognitive decline of aged dogs represents serious medical and social problem. Increasing number of dogs older than 7 years is leading to a higher prevalence of CCDS worldwide [2]. The aging profile of dogs varies 
Table 1 Distribution of dogs throughout selected categories and stages of canine dementia

\begin{tabular}{|c|c|c|c|c|c|c|c|c|c|c|c|}
\hline \multirow[t]{2}{*}{ Categories } & \multirow[t]{2}{*}{$\begin{array}{l}\text { Total number } \\
\text { of dogs }\end{array}$} & \multicolumn{2}{|l|}{ Weight } & \multicolumn{2}{|l|}{ Nutrition } & \multicolumn{2}{|l|}{ Sex } & \multicolumn{2}{|c|}{ Dogs' housing } & \multicolumn{2}{|c|}{$\begin{array}{l}\text { Reproductive } \\
\text { status }\end{array}$} \\
\hline & & Over 15 kg & Below 15 kg & $\begin{array}{l}\text { Uncontrolled } \\
\text { diet }\end{array}$ & $\begin{array}{l}\text { Controlled } \\
\text { diet }\end{array}$ & Females & Males & Inside & Outside & Neutered & Entire \\
\hline $\begin{array}{l}\text { Normal } \\
\text { ageing }\end{array}$ & 56 & 31 & 25 & 21 & 35 & 30 & 26 & 34 & 22 & 43 & 13 \\
\hline $\begin{array}{l}\text { Mild cognitive } \\
\text { impairment }\end{array}$ & 80 & 38 & 42 & 31 & 49 & 37 & 43 & 52 & 28 & 64 & 16 \\
\hline $\begin{array}{l}\text { Moderate } \\
\text { cognitive } \\
\text { impairment }\end{array}$ & 49 & 23 & 26 & 30 & 19 & 19 & 30 & 32 & 17 & 38 & 11 \\
\hline $\begin{array}{l}\text { Severe cogni- } \\
\text { tive impair- } \\
\text { ment }\end{array}$ & 30 & 11 & 19 & 20 & 10 & 13 & 17 & 19 & 11 & 22 & 8 \\
\hline
\end{tabular}

Table 2 The results of statistical analyses of the risk factors-weight, nutrition, sex, dogs' housing and reproductive state for canine dementia

\begin{tabular}{|c|c|c|c|c|c|c|c|c|c|c|}
\hline Variable (group 1 vs group 2) & $\mathrm{n}_{1}$ & $\mathrm{~N}_{1}$ & $\mathbf{n}_{2}$ & $\mathrm{~N}_{2}$ & OR & sd (OR) & LB & UB & Z-statistics & $P$ value \\
\hline Weight OR (under 15 kg vs over 15 kg) & 45 & 112 & 34 & 103 & 1.36 & 0.21 & 0.78 & 2.38 & 1.0879 & 0.1383 \\
\hline Nutrition OR (uncontrolled vs controlled diet) & 50 & 102 & 29 & 113 & 2.79 & 0.82 & 1.57 & 4.94 & 3.5006 & 0.0002 \\
\hline Sex OR (males vs females) & 47 & 116 & 32 & 99 & 1.43 & 0.20 & 0.81 & 2.50 & 1.2401 & 0.1075 \\
\hline Dogs' housing OR (outside vs inside) & 51 & 137 & 28 & 78 & 1.06 & 0.28 & 0.59 & 1.89 & 0.1943 & 0.4230 \\
\hline Reproductive state OR (neutered vs entire dogs) & 60 & 167 & 19 & 48 & 0.86 & 0.39 & 0.44 & 1.65 & -0.4627 & 0.3218 \\
\hline
\end{tabular}

$n_{1}$ the number of moderate and severe cognitive impaired dogs in group 1 (weight below $15 \mathrm{~kg}$, nutrition-uncontrolled diet, males, housing inside, neutered), $n_{2}$ the number of moderate and severe cognitive impaired dogs in group 2 (weight above $15 \mathrm{~kg}$, nutrition-controlled diet, females, housing outside, entire), $N_{1}, N_{2}$ total number of dogs in group 1 and group 2, OR odds ratio, sd (OR) standard deviation of odds ratio, LB lower bound, UB upper bound of Wald $95 \%$ empirical confidence interval $(\mathrm{Cl})$ for $\mathrm{OR}$, Z-statistics two-sample $Z$-test about log $\mathrm{OR}$ ( $P$ values related to statistically significant results are highlighted in italics; the bounds of $\mathrm{Cl}$ are back transformed bounds of $\mathrm{Cl}$ for log $\mathrm{OR}$ )

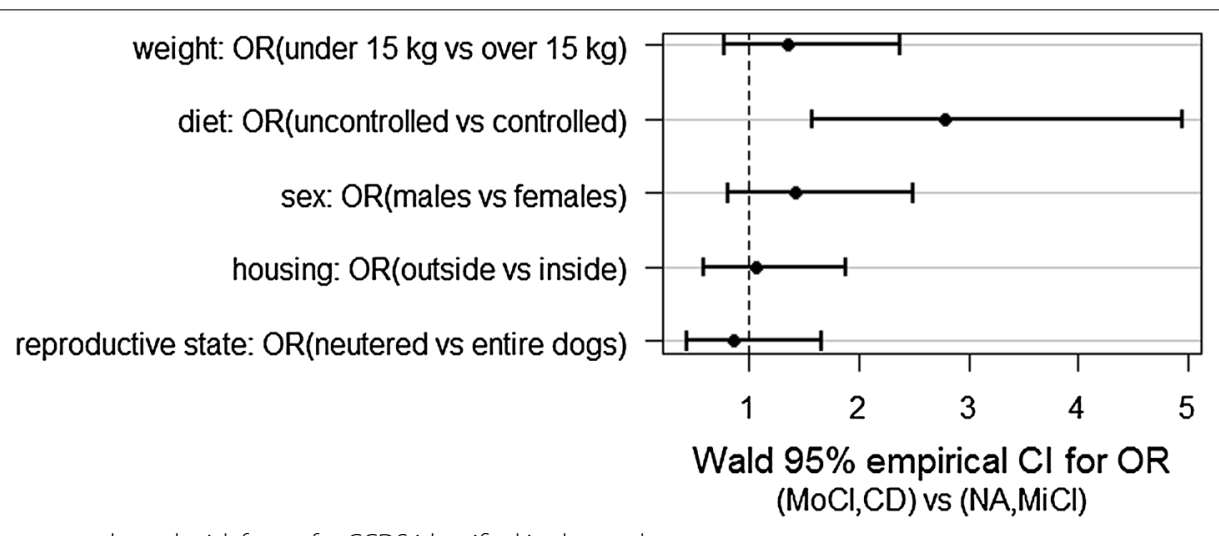

Fig. 2 Nutrition represents the only risk factor for CCDS identified in the study

between breeds, generally smaller dogs can live up to 10-14 years, while medium and large sized breeds live up to 8 years [14]. Today, dogs are generally living longer than previously thanks to increasing knowledge about nutritional needs and advances in veterinary medicine. Increasing life span does not equal increasing life quality as shown by higher prevalence of age-related disorders or healthy problems such as arthritis and joint problems, prostate enlargement, urinary incontinence, constipation, hearing and vision loss.

Several studies have shown that the prevalence of CCDS raises with increased age $[4,5,8,9]$. However, 
Table 3 The prevalence of CCDS

\begin{tabular}{llll}
\hline Current paper & & & \\
& $8-11$ years & $11-13$ years & Over 13 years \\
Small breed (\%) & 13 & 41 & 87 \\
$\quad$ Medium/large breed (\%) & 16 & 55 & 100 \\
Salvin et al. [9] & & & \\
$8-10$ years & $10-12$ years & $12-14$ years & Over 14 years \\
$3.4 \%$ & $5 \%$ & $23.3 \%$ & $41 \%$ \\
Azkona et al. [5] & & & \\
& $9-11$ years & $12-14$ years & $15-17$ years \\
Small breed (\%) & 22.7 & 32.7 & 50.0 \\
Medium/large breed (\%) & 10.3 & 26.9 & 40 \\
\hline
\end{tabular}

telephone interviews used in some studies cannot exclude various medical causes of CCDS and thus the prevalence of CCDS can be overestimated. To strengthen the design of the study, we combined structured interviews with pet owners with direct clinical evaluation. Previously, Salvin et al. [9] showed that the prevalence of CCDS increased with age. Similarly, Azkona et al. [5] demonstrated increased prevalence of CCDS in both small breeds and medium/large breeds. In contrast, we observed greater prevalence of CCDS in medium/large sized breeds compared with small sized breeds in the age group of 11-13 years, while no difference was observed in the age group of 8-11 years. Our findings are in accordance with a previous study that focused on the relationship between body size and life span [14]. The researchers clearly demonstrated that while there was no clear correlation between body size and the onset of senescence, there was a strong positive relationship between size and aging rate. Finally, they concluded that dogs of large breeds died younger than small breed dogs, mainly because they aged more quickly. We plan to increase the total number of dogs to identify whether some of the breeds are more vulnerable to CCDS than the others.

The increasing incidence of CCDS highlights the importance of identification of putative aetiological risk factors. Unfortunately, knowledge regarding risk factors of CCDS is still quite limited and some data are rather contradictory, e.g., one study found that sex and size of breeds had no association with CCDS [7], while another study indicated that small sized dogs and females had a greater risk of developing signs of CCDS [5]. Another proposed risk factor was the reproductive state. Azkona et al. [5] demonstrated a higher risk of CCDS in neutered female and male dogs in comparison to intact dogs. Similarly, Hart [6] showed that sexually intact male dogs were significantly less likely than neutered dogs to progress from mild impairment to severe impairment. Surprisingly, our data did not support the contribution of sex, reproductive status and weight to the disease progression. We also did not find any correlation between dogs' housing and cognitive decline. In case of dogs' housing and reproduction state, we are aware of the fact, that the number of dogs in each group (neutered vs entire, outside vs inside) is not equal. In our study, we recruited much more neutered dogs than intact dogs and the majority of dogs lived inside of houses or flats. This uneven distribution may have influenced the outcome of the study.
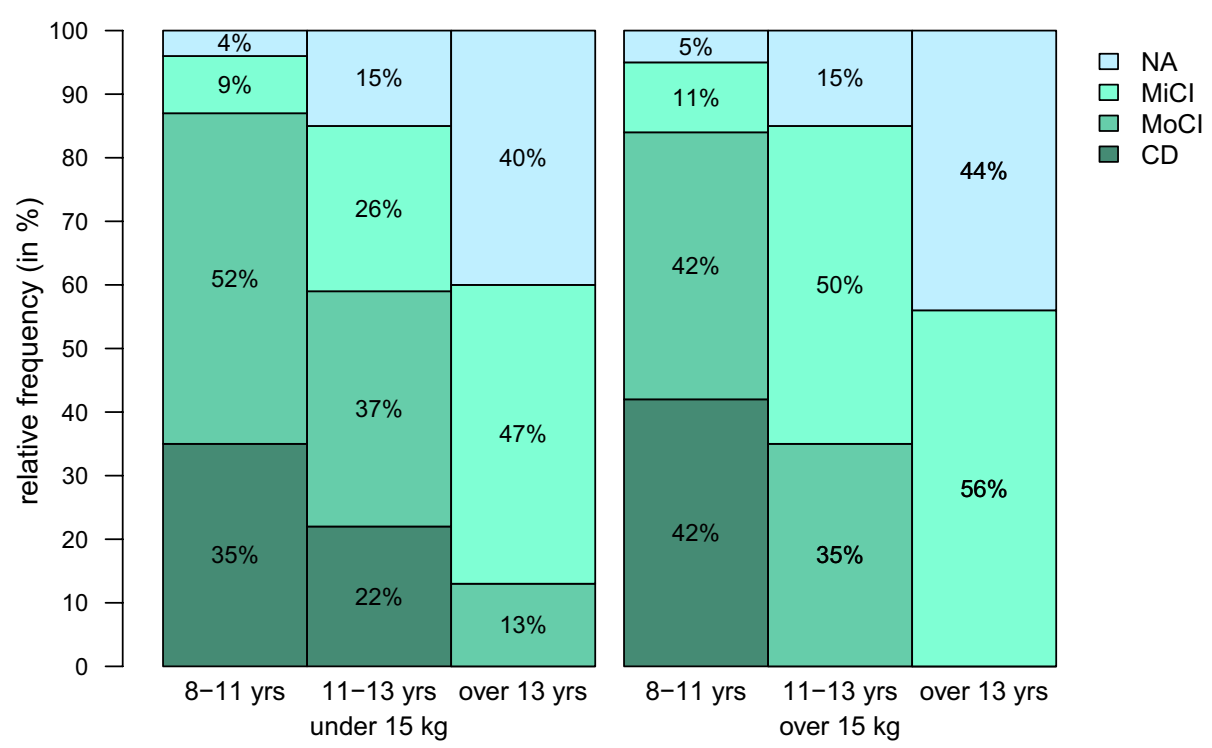

Fig. 3 The prevalence of CCDS. Barplots demonstrate that the prevalence of CCDS increases with age. NA normal ageing, MiCl mild cognitive impairment, $\mathrm{MoCl}$ moderate cognitive impairment, $C D$ severe cognitive impairment 
On the other hand, we found for the first time that nutrition may represent an important factor for development of CCDS. We defined two groups of diets as proposed by Hand et al. [15]: controlled diet and uncontrolled food. In this study, controlled diet is characterized as high-quality commercial food purchased for the size of the breed or age or healthy status demands (Hill's, Royal Canine, Specific etc.). Uncontrolled diet represents kitchen waste, unspecified feed mixture or lowquality commercial food, which is not purchased for the size of the breed or age or healthy status demands. Our study revealed that uncontrolled diets may increase a dog's risk of developing CCDS. Based on our results we suggest that controlled diets could be more protective against cognitive damage than homemade or mixed diets. Our findings may explain why nutritional manipulation through the use of special diets and dietary supplements may be beneficial for treatment and prevention of CCDS [16]. We suggest that CCDS is an aging-related multifactorial disorder that may be modified by a properly balanced diet.

We are aware of strengths and weaknesses of our study. While this study included only a small number of subjects and conducted in one clinic in Slovakia, and therefore, may represent regional and/or cultural group, the results are consistent with several independent nutritional studies showed that controlled diet can significantly improve, or slow the decline of learning and memory in aged dogs $[17,18]$. Moreover, it has been demonstrated that antioxidants and mitochondrial cofactors, and long-term supplementation with medium-chain triglycerides improve cognitive function in aged dogs [19].

\section{Conclusions}

We demonstrated that the prevalence of CCDS almost tripled in small dogs and quadrupled in medium/large dogs, within the age period from 8 to 13 years. We also confirmed previous studies showing that age represent the main risk factors for CCDS. We found that nutrition may represent important factor in the development of CCDS at least in the dog population of Slovakia. Further studies are warranted to support this finding. This study further highlights the urgent need for effective preventative strategies and therapeutic approaches.

\section{Abbreviations}

Alb: albumin; ALT: alanine aminotransferase; ALP: alkaline phosphatase; AST: aspartate aminotransferase; CADES: canine dementia scale; CCDS: canine cognitive dysfunction syndrome; Chol: cholesterol; Crea: creatine; DISHA: disorientation, interaction changes, sleep/wake disturbances, house soiling and activity changes; ECG: electrocardiography; Glu: glucose; HCT: haematocrit; LIP: lipase; RBC: red blood cells; HGB: haemoglobin; MiCl: mild cognitive impairment; $\mathrm{MCl}$ : human mild cognitive impairment; $\mathrm{MCH}$ : mean corpuscular hemoglobin; MCHC: mean corpuscular hemoglobin concentration; MCV: mean corpuscular volume; MoCl: moderate cognitive impairment; MPV: mean
}

platelet volume; NA: normal ageing; nRBCs: nucleated red blood cells; OD: odds ratio; PCT: plateletcrit; PAMS: amylase; PDW: platelet distribution width; RDW: red cell distribution width; TP: total protein.

\section{Authors' contributions}

SK performed the statistical analyses and performed the final correction of the manuscript. AM and JF performed the behavioural examination of dogs. NZ and $\mathrm{MN}$ analysed and interpreted data. All authors participated in the writing of the manuscript. All authors read and approved the final manuscript.

\section{Author details}

${ }^{1}$ Department of Mathematics and Statistics, Masaryk University, Kotlarska 2, Brno, Czech Republic. ${ }^{2}$ University of Veterinary Medicine and Pharmacy, Komenskeho 73, Kosice, Slovak Republic. ${ }^{3}$ Institute of Neuroimmunology, Slovak Academy of Sciences, Dubravska cesta 9, Bratislava, Slovak Republic. ${ }^{4}$ Institute of Neuroimmunology, No., Dvorakovo Nabrezie 45, Bratislava, Slovak Republic.

\section{Acknowledgements}

This work is dedicated to Prof. Marian Kozak, the father of the cognitive research in Slovak veterinary medicine. This work was supported by research Grants APVV 0206-11 and EU structural fund 26240220046

\section{Competing interests}

The authors declare that they have no competing interests.

Received: 5 September 2015 Accepted: 9 February 2016

Published online: 29 February 2016

\section{References}

1. Landsberg GM, Nichol J, Araujo JA. Cognitive dysfunction syndrome: a disease of canine and feline brain aging. Vet Clin North Am Small Anim Pract. 2012;42:749-68.

2. Bosch MN, Pugliese M, Gimeno-Bayón J, Rodríguez MJ, Mahy N. Dogs with cognitive dysfunction syndrome: a natural model of Alzheimer's disease. Curr Alzheimer Res. 2012;9:298-314.

3. Sanabria CO, Olea F, Rojas M. Cognitive dysfunction syndrome in senior dogs. In: Kishore U, editor. Neurodegenerative diseases, 2013. p. 615-628.

4. Neilson JC, Hart BL, Cliff KD, RuehI WW. Prevalence of behavioral changes associated with age-related cognitive impairment in dogs. J Am Vet Med Assoc. 2001;218:1787-91.

5. Azkona G, García-Belenguer S, Chacón G, Rosado B, León M, Palacio J. Prevalence and risk factors of behavioural changes associated with age-related cognitive impairment in geriatric dogs. J Small Anim Pract. 2009;50:87-91.

6. Hart BL. Effect of gonadectomy on subsequent development of age-related cognitive impairment in dogs. J Am Vet Med Assoc. 2001;219:51-6.

7. Fast R, Schütt T, Toft N, Møller A, Berendt M. An observational study with long-term follow-up of canine cognitive dysfunction: clinical characteristics, survival, and risk factors. J Vet Int Med. 2013;27:822-9.

8. Osella MC, Re G, Odore R, Girardi C, Badino P, Barbero R, Bergamasco L. Canine cognitive dysfunction syndrome: prevalence, clinical signs and treatment with a neuroprotective nutraceutical. Appl Anim Beh Sci. 2007:105:297-310.

9. Salvin HE, McGreevy PD, Sachdev PS, Valenzuela MJ. Under diagnosis of canine cognitive dysfunction: a cross-sectional survey of older companion dogs. Vet J. 2010;184:277-81

10. Madari A, Farbakova J, Katina S, Smolek T, Novak P, Weissova T, Novak M, Zilka N. Assessment of severity and progression of canine cognitive dysfunction syndrome using CAnine DEmentia Scale (CADES). Appl Anim Behav Sci. 2015;171:138-45.

11. Michell AR. Longevity of British breeds of dog and its relationships with sex, size, cardiovascular variables and disease. Vet Rec. 1999;145:625-9.

12. R Development Core Team. R: A language and environment for statistical computing. Vienna: R Foundation for Statistical, Computing; 2014

13. Lachin JM. Biostatistical methods. The assessment of relative risks. Hoboken: Wiley \& Sons; 2011. 
14. Kraus C, Pavard S, Promislow DE. The size-life span trade-off decomposed: why large dogs die young. Amer Nat. 2013;181:492-505.

15. Hand MS, Thatcher CD, Remillard RL, Roudebush P. Small animal clinical nutrition. 5th ed. Marceline: Walsworth publishing Company; 2010.

16. Zicker SC. Cognitive and behavioral assessment in dogs and pet food market applications. Prog Neuropsychopharmacol Biol Psychiatry. 2005;29:455-9.
17. Head E, Rofina J, Zicker S. Oxidative stress, aging, and central nervous system disease in the canine model of human brain aging. Vet Clin North Am Small Anim Pract. 2008;38:167-78.

18. Pan Y. Enhancing brain functions in senior dogs: a new nutritional approach. Top Companion Anim Med. 2011;26:10-6.

19. Manteca X. Nutrition and behavior in senior dogs. Top Companion Anim Med. 2011;26:33-6.
Submit your next manuscript to BioMed Central and we will help you at every step:

- We accept pre-submission inquiries

- Our selector tool helps you to find the most relevant journal

- We provide round the clock customer support

- Convenient online submission

- Thorough peer review

- Inclusion in PubMed and all major indexing services

- Maximum visibility for your research

Submit your manuscript at www.biomedcentral.com/submit
(O) BioMed Central 